\title{
A Low-Dropout Regulator for SoC With $Q$-Reduction
}

\author{
Sai Kit Lau, Member, IEEE, Philip K. T. Mok, Senior Member, IEEE, and Ka Nang Leung, Member, IEEE
}

\begin{abstract}
A low-dropout regulator for SoC, with an advanced $Q$-reduction circuit to minimize both the on-chip capacitance and the minimum output-current requirement down to $100 \mu \mathrm{A}$, is introduced in this paper. The idea has been implemented in a standard $0.35-\mu \mathrm{m}$ CMOS technology $\left(V_{\mathrm{THN}} \approx 0.55 \mathrm{~V}\right.$ and $\left.\left|V_{\text {THP }}\right| \approx 0.75 \mathrm{~V}\right)$. The required on-chip capacitance is reduced to $6 \mathrm{pF}$, comparing to $25 \mathrm{pF}$ for the case without $Q$-reduction circuit. From the experimental results, the proposed regulator-circuit implementation enables voltage regulation down to a 1.2-V supply voltage, and a dropout voltage of $200 \mathrm{mV}$ at $100-\mathrm{mA}$ maximum output current.
\end{abstract}

Index Terms-Low-dropout regulator, power management, $Q$-reduction.

\section{INTRODUCTION}

$\mathbf{P}$ OWER management utilizing multiple local on-chip voltage regulators to power-up system sub-blocks individually, as shown in Fig. 1, is a very promising approach in system-on-a-chip (SoC) development [1]. In addition to the advantage of tailor-making supply voltages for optimizing performance of different circuits separately, power-line cross-talk problem can also be lessened. This approach is especially suitable for portable systems, since lower usage of PCB space and fewer off-chip components are advantageous to production. Of all the types of voltage regulators, low-dropout regulator (LDO) is regarded as a suitable choice for local on-chip voltage regulation in $\mathrm{SoC}$, due to its fast transient response and low-noise advantages. However, an off-chip capacitor at LDO output, generally about $0.47 \mu \mathrm{F}$ to $10 \mu \mathrm{F}$ for a 200-mA $\mathrm{LDO}$, is necessary to provide good dynamic performance and simultaneously ensure the regulator's stability [2]-[6]. On-chip capacitance, for example, the 0.6-nF decoupling capacitance in [6], consumes huge silicon area and is undoubtedly an obstacle in the $\mathrm{SoC}$ development.

Currently, many researchers and companies have been developing LDOs which do not need off-chip load capacitor for stability and performance. Many approaches, such as using nonstandard technology-based approach, (for example, DMOS in

Manuscript received March 6, 2006; revised October 27, 2006. This work was supported by the Research Grant Council of Hong Kong SAR Government, China, under Project HKUST6150/03E.

S. K. Lau was with the Department of Electronic and Computer Engineering, The Hong Kong University of Science and Technology, Hong Kong. He is currently with Pericom Technology Inc., Hong Kong Science Park, Pak Shek Kok, Hong Kong (e-mail: eedennis@ieee.org).

P. K. T. Mok is with the Department of Electronic and Computer Engineering, The Hong Kong University of Science and Technology, Clear Water Bay, Kowloon, Hong Kong (e-mail: eemok@ece.ust.hk).

K. N. Leung was with the Department of Electronic and Computer Engineering, The Hong Kong University of Science and Technology, Clear Water Bay, Hong Kong. He is currently with the Department of Electronic Engineering, The Chinese University of Hong Kong, Shatin, Hong Kong (e-mail: knleung@ee.cuhk.edu.hk).

Digital Object Identifier 10.1109/JSSC.2006.891496

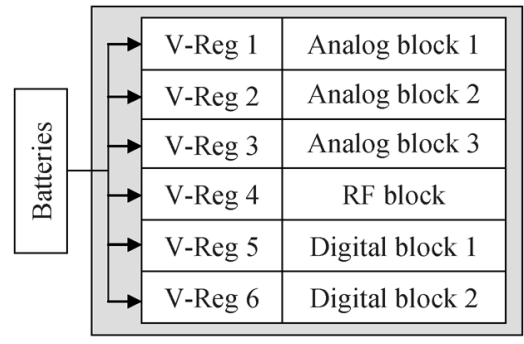

Fig. 1. An example, simplified power distribution of SoC.

[7]), and circuit-level-based pole-splitting approach [8], have been proposed with good performance. The conceptual figure of a LDO, based on pole-splitting method, is shown in Fig. 2. The LDO is viewed as a three-stage amplifier compensated by pole-splitting frequency compensation. The power dissipated at the power transistor increase its transconductance $\left(g_{m p}\right)$. A higher $g_{m p}$, due to moderate output current, results in higher nondominant complex-pole frequencies. When the nondominant complex poles locate far higher than the unity-gain frequency (UGF) in the open-loop frequency response, the LDO is stable [9].

However, as illustrated in Fig. 3, when the output current is low, the nondominant complex poles have a large $Q$ (or small damping factor) and locate near UGF. A large $Q$ causes a sharper phase change at the angular corner frequency. The LDO will then be unstable due to the magnitude peaking near UGF [9]. The minimum output current requirement of the pole-splitting-based LDO is in the range of several milli-ampere and should be further reduced for voltage regulation in a wider current range. One method to reduce the minimum output current level is to increase the on-chip compensation capacitance for lowering the UGF, since the UGF is inversely proportional to the compensation capacitance, as shown in Fig. 3 [9]. However, the larger on-chip capacitance consumes more chip area, and therefore, is not a good solution. Further development, focusing on minimizing the output current condition by advanced $Q$-reduction methods to control the nondominant complex poles, is one of the design directions of LDOs for SoC applications.

In this paper, a LDO targeted for SoC will be proposed. The key feature is to reduce required on-chip capacitance with the proposed $Q$-reduction circuit. Advanced circuit implementation of the $Q$-reduction circuit will then be introduced and discussed. Finally, experimental results will be given to verify the theory.

\section{Proposed LDO Structure}

The schematic of the proposed LDO is shown in Fig. 4. M01, M02, M03, and M04 form the first stage and the proposed current buffer (formed by M03 and M04 and $C_{c f}$ ), while M05, M06, M07, and M08 form the second noninverting gain stage. MPT is the power pMOSFET forming the third stage, and $C_{g d}$ 


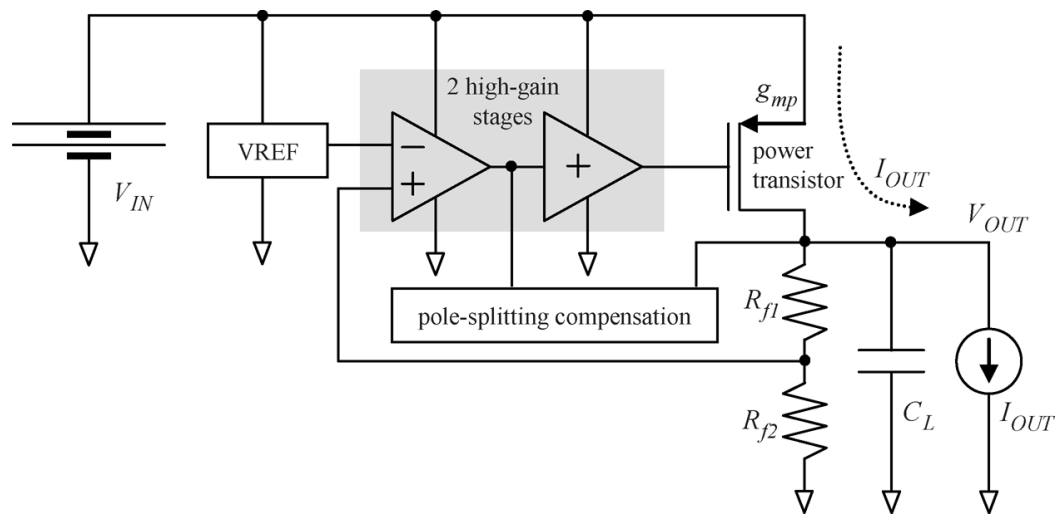

Fig. 2. Conceptual structure of the LDO in [8].

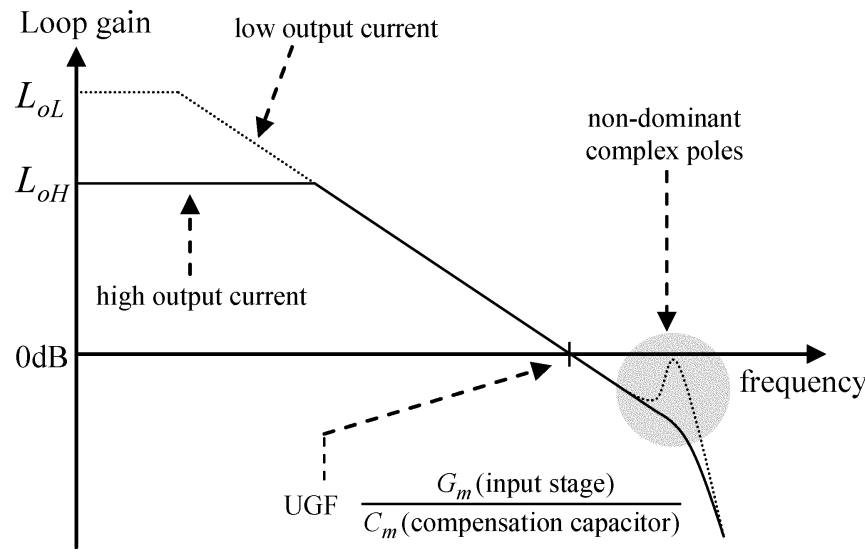

Fig. 3. Loop gains of pole-splitting-based LDO at different output currents.

is its gate-drain parasitic capacitance. M01, M03, and M08 form the feed-forward transconductance stage. $C_{m 1}$ and $C_{c f}$ are the required on-chip capacitance of the proposed structure. $R_{f 1}$ and $R_{f 2}$ construct the feedback resistive network. $R_{L}$ and $C_{3}$ model the equivalent load resistance and load capacitance at the power line. Fig. 4 shows that the bias current of the current buffer is, in fact, the bias current of the input stage. The high-gain property of the amplifier enforces the bias current of M03 and M04 to be nearly equal. Therefore, the proposed circuit implementation is simpler and consumes less power. Moreover, the small-signal current signals from input stage and from $C_{c f}$ can be summed together to generate $v_{1}$.

A smaller $g_{m c f}$, which is the transconductance of M03, is needed to reduce $Q$. This is achieved by a small aspect size ratio of M03 and M04. This design requirement helps to reduce the random offset voltage of the input stage due to the mismatch of the threshold voltage of pMOS so that the LDO output voltage has less error due to process variations in mass production [10]. The feed-forward transconductance stage is formed by
M01, M03 and M08. Both M06 and M08 form a push-pull stage such that the gate capacitance of the power pMOSFET can be charged and discharged more effectively for fast load transient responses.

The proposed LDO in Fig. 4 can be modeled by Fig. 5 in open-loop domain. The structure is basically composed of three gain stages (not including the $Q$-reduction circuit), where the third stage is the power pMOSFET in common-source configuration. Since the transistor size of the power pMOSFET is large, its gate-drain capacitance $\left(C_{g d}\right)$ is also large and should be included for detailed analysis. The corresponding transconductance, output resistance and lumped output parasitic capacitance of each stage are denoted by $g_{m i}, R_{i}$ and $C_{i}$ (for $i=1,2$, and 3 ), respectively. A compensation capacitor $C_{m 1}$ enables pole-splitting effect [9]. The proposed $Q$-reduction circuit is formed by $C_{c f}$ and a current buffer (where the transconductance, input resistance, and input capacitance are $g_{m c f}, R_{c f} \approx 1 / g_{m c f}$, and $C_{p}$, respectively). In this analysis, the current buffer is assumed to have ideal frequency response, which is valid in LDO design since the parasitic capacitances due to the current buffer are much smaller than those from the power transistor. As will be discussed, a feed-forward transconductance stage $\left(g_{m f 1}\right)$ is used to generate a left-half-plane (LHP) zero to improve both LDO stability and slewing at the gate of the power pMOSFET. Moreover, $C_{3}$ is the parasitic capacitance due to the power line in $\mathrm{SoC}$ and is generally larger than the internal nodal capacitances $C_{1}, C_{2}$, and $C_{p}$. The signal transfer function of the proposed LDO structure, regarded as loop-gain frequency response of a feedback circuit $L(s)$, is given by (1), shown at the bottom of the page. Since the output current of a LDO will change (so as $g_{m 3}$ will change), the stability of the proposed LDO should be considered at different load conditions. In this analysis, the stability of LDO is considered when the feedback factor defined by the feedback resistors is 1 (i.e., $V_{\mathrm{OUT}}=V_{\mathrm{REF}}$ ). This guarantees that the proposed LDO is stable when $V_{\mathrm{OUT}}>V_{\mathrm{REF}}$ in

$L(s)=\frac{v_{o}(s)}{v_{i}(s)} \approx \frac{-g_{m 1} g_{m 2} g_{m 3} R_{1} R_{2} R_{3}\left\{1+s\left(C_{c f} R_{c f}+\frac{C_{m 1} g_{m f 1}}{g_{m 1} g_{m 2}}-\frac{C_{g d}}{g_{m 3}}\right)-s^{2}\left[\frac{C_{m 1}\left(C_{g d}+C_{2}\right)}{g_{m 2} g_{m 3}}+\frac{C_{g d} C_{c f} R_{c f}}{g_{m 3}}\right]\right\}}{\left(1+s C_{m 1} g_{m 2} g_{m 3} R_{1} R_{2} R_{3}\right) \cdot\left[1+s \frac{C_{m 1} C_{g d}\left(g_{m 3}-g_{m 2}\right)+C_{c f} C_{3} g_{m 2}+C_{m 1} C_{c f} g_{m 2} g_{m 3} R_{c f}}{C_{m 1} g_{m 2} g_{m 3}}+s^{2} \frac{\left(C_{g d}+C_{2}+C_{c f}\right) C_{3}}{g_{m 2} g_{m 3}}\right]}$ 


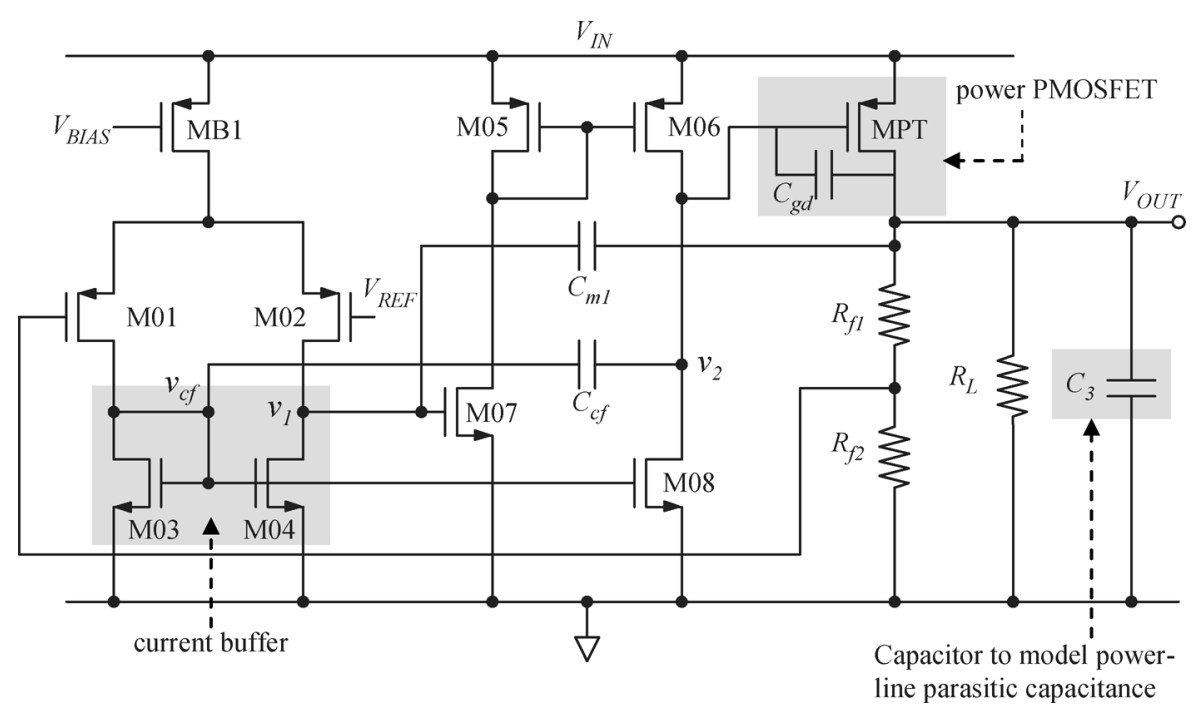

Fig. 4. Schematics of the proposed CMOS LDO.

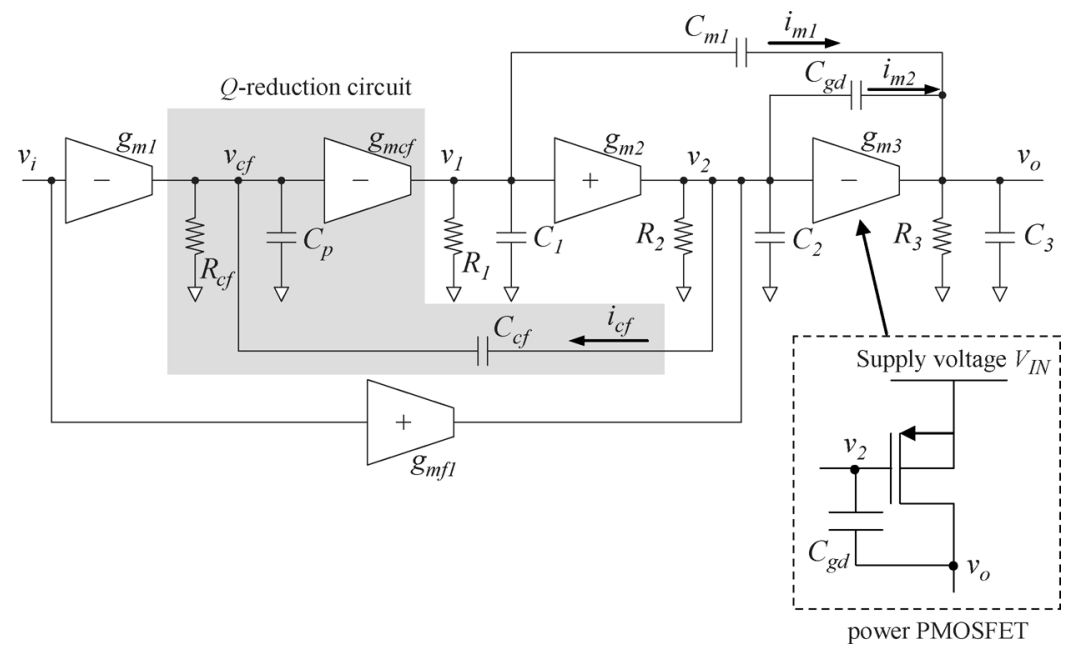

Fig. 5. Proposed LDO structure (open loop).

general operations. There are three cases to consider when $C_{3}$ is due to the parasitic capacitance of the power line and is not large.

Case 1: Moderate to Maximum Output Current (i.e., $g_{m 3}$ is Much Larger Than $g_{m 1}$ and $g_{m 2}$ ): The transfer function in (1) is approximated as given in (2), shown at the bottom of the page. The second-order function at the denominator of (2) gives two separate poles when the condition $\left(4\left(C_{g d}+C_{2}+\right.\right.$ $\left.\left.C_{c f}\right) \cdot C_{3}\right) / g_{m 3} \leq\left(C_{g d}+C_{c f}\right)^{2} / g_{m 2}$ holds. That condition happens when the output current is high so that $g_{m 3}$ is very large. At this condition, there are three poles and one zero, given by $p_{1}=1 / C_{m 1} g_{m 2} g_{m 3} R_{1} R_{2} R_{3}, p_{2}=g_{m 2} /\left(C_{g d}+C_{c f}\right)$, $p_{3}=\left(\left(C_{g d}+C_{c f}\right) \cdot g_{m 3}\right) /\left(\left(C_{g d}+C_{2}+C_{c f}\right) \cdot C_{3}\right)$ and $z_{1}=g_{m 1} g_{m 2} / C_{m 1} g_{m f 1}$, respectively. With a large $g_{m 3}, p_{3}$ locates at a high frequency and has no effect on LDO stability. When $z_{1}$ is used to cancel $p_{2}$, there is effectively one pole and the proposed LDO is stable for any resistive feedback factor with a theoretical phase margin of $90^{\circ}$.

Case 2: Low to Moderate Load Current (i.e., $g_{m 3}$ is Larger Than $g_{m 1}$ and $g_{m 2}$ ): The transfer function is the same as (1).

$$
L(s) \approx \frac{-g_{m 1} g_{m 2} g_{m 3} R_{1} R_{2} R_{3} \cdot\left(1+\frac{s C_{m 1} g_{m f 1}}{g_{m 2} g_{m 3}}\right)}{\left(1+s C_{m 1} g_{m 2} g_{m 3} R_{1} R_{2} R_{3}\right)\left[1+s \frac{\left(C_{g d}+C_{c f}\right)}{g_{m 2}}+s^{2} \frac{\left(C_{g d}+C_{2}+C_{c f}\right) C_{3}}{g_{m 2} g_{m 3}}\right]}
$$


There is a pair of complex poles since the coefficients of (1) have the following relationship when $g_{m 3}$ is not much larger than $g_{m 1}$ and $g_{m 2}$ :

$$
\begin{gathered}
{\left[\frac{C_{m 1} C_{g d}\left(g_{m 3}-g_{m 2}\right)+C_{c f} C_{3} g_{m 2}+C_{m 1} C_{c f} g_{m 2} g_{m 3} R_{c f}}{C_{m 1} g_{m 2} g_{m 3}}\right]^{2}} \\
\leq 4 \frac{\left(C_{g d}+C_{2}+C_{c f}\right) C_{3}}{g_{m 2} g_{m 3}} .
\end{gathered}
$$

By solving (1), there are one pole $\left(p_{1}=\right.$ $\left.1 / C_{m 1} g_{m 2} g_{m 3} R_{1} R_{2} R_{3}\right)$, one pair of complex poles, and one zero $\left(z_{1}=g_{m 1} g_{m 2} / C_{m 1} g_{m f 1}\right)$. The pole frequency of the complex poles is given by

$$
\omega_{O} \approx \sqrt{\frac{g_{m 2} g_{m 3}}{\left(C_{g d}+C_{2}\right) \cdot C_{3}}}
$$

and its $Q$ is given by (5), shown at the bottom of the page, where $k_{1}, k_{2}, k_{3}$, and $k_{4}$ are constants dependent on load-current level. From (4), a higher output current, hence a larger $g_{m 3}$, results in a higher $\omega_{O}$ and the stability of the proposed LDO will be improved. More importantly, from (5), the proposed $Q$-reduction circuit decreases $Q$ linearly by a larger $C_{c f}$ and a smaller $g_{m c f}$ of the current buffer. Therefore, the minimum output current of the proposed LDO can be reduced effectively by controlling $C_{c f}$ and $g_{m c f}$ separately. As a reference, for the same circuit structure and transistor parameters $\left(g_{m 1}=120 \mu \mathrm{A} / \mathrm{V}, g_{m 2}=480 \mu \mathrm{A} / \mathrm{V}, g_{m 3}=1.5 \mathrm{~mA} / \mathrm{V}\right.$, $\left.C_{g d}=6.4 \mathrm{pF}, C_{3}=100 \mathrm{pF}\right)$, the $Q$ factors with $Q$-reduction technique $\left(C_{m 1}=5 \mathrm{pF}\right.$ and $\left.C_{c f}=1 \mathrm{pF}\right)$ and without $\left(C_{m 1}=25 \mathrm{pF}\right.$ and $C_{c f}=0$, note that in this case, a larger $C_{m 1}$ is needed for stable loop gain) is 2.2 and 7.4, respectively. There is about 3.4 times reduction on the $Q$ factor and hence a smaller $C_{m 1}$ can be used when the proposed $Q$-reduction technique is employed. The phase margin (PM) of the LDO in this case can be evaluated by [11], [12]

$$
\begin{aligned}
\mathrm{PM}= & 180^{\circ}-\tan ^{-1}\left(\frac{\mathrm{UGF}}{p_{1}}\right) \\
& -\tan ^{-1}\left\{\frac{\mathrm{UGF} / \omega_{O}}{Q\left[1-\left(\mathrm{UGF} / \omega_{O}\right)^{2}\right]}\right\}+\tan ^{-1}\left(\frac{\mathrm{UGF}}{z_{1}}\right) \\
= & 90^{\circ}-\tan ^{-1}\left\{\frac{\mathrm{UGF} / \omega_{O}}{Q\left[1-\left(\mathrm{UGF} / \omega_{O}\right)^{2}\right]}\right\} \\
& +\tan ^{-1}\left(\frac{\mathrm{UGF}}{z_{1}}\right)
\end{aligned}
$$

where $\mathrm{UGF}=g_{m 1} / C_{m 1}$. From (6), there are a few important implications for design.
1) The critical phase reduction is due to $\omega_{O}$. Therefore, a lower UGF by a larger $C_{m 1}$ can help to increase the phase margin.

2) A smaller $g_{m c f}$ and a larger $C_{c f}$ help to reduce Q, as shown in (5). This can help to reduce the negative phase shift for more phase margin.

3) The parameter $g_{m f 1}$ is very useful to control the position of $z_{1}$ for positive phase shift. Since, according to (4), $\omega_{O}$ is low when $g_{m 3}$ is low (or light load). The position of $z_{1}$, in terms of $g_{m f 1}$, should be designed in light-load condition.

Case 3: Very Light Output Current: When the load current is very small, the small $g_{m 3}$ will result in an unstable LDO. The boundary between stable and unstable regions of the proposed LDO is addressed here. It is well-known that right-half-plane (RHP) pole causes unstable negative feedback system [10]. From the denominator of (1), RHP poles can be avoided when the $s$ term in the second-order function is positive. Therefore, the necessary condition is given by

$C_{m 1} C_{g d}\left(g_{m 3}-g_{m 2}\right)+C_{c f} C_{3} g_{m 2}+C_{m 1} C_{c f} g_{m 2} g_{m 3} R_{c f}>0$.

By rearranging the term, the minimum $g_{m 3}$ is

$$
g_{m 3(\min )}=\frac{\left(\frac{C_{c f}+C_{3}}{C_{m 1}+C_{g d}}\right) \cdot g_{m 2}}{1-\left(\frac{C_{c f}}{C_{g d}}\right) \cdot g_{m 2} R_{c f}} .
$$

Therefore, the minimum load current that the LDO is stable can be found indirectly from $g_{m 3(\mathrm{~min})}$. In general, it requires a current higher than the bias current of the resistive feedback network that passing through the power pMOSFET.

A loop-gain simulation of the proposed LDO in Fig. 6 has been performed to study the stability. The simulation is with a BSIM3v3 0.35- $\mu$ m CMOS model from Austria Mikro System Group, Austria (AMS). The capacitance $C_{3}$ at power line is assumed to be $100 \mathrm{pF}$ in the simulation. From the simulation results in Fig. 6, the stability of the proposed LDO is better when the output current is higher, as expected according to the stated theory. The nondominant poles locate separately in frequency domains at higher output current, while a pair of complex poles with a higher $Q$ is generated at lower output current. Based on the proposed structure and implementation, the minimum output current is about $100 \mu \mathrm{A}$ when an on-chip $C_{c f}$ of only $1 \mathrm{pF}$ is used. Moreover, the effect due to process and temperature variations is also studied by simulations. The proposed LDO is loaded with a current of $100 \mu \mathrm{A}$. Corner transistor models provided by the foundry are used to investigate the impact to sta-

$$
\begin{aligned}
Q & =\sqrt{\frac{\left(C_{g d}+C_{2}\right) C_{3}}{g_{m 2} g_{m 3}}} \cdot\left[\frac{C_{m 1} g_{m 2} g_{m 3}}{C_{m 1} C_{g d}\left(g_{m 3}-g_{m 2}\right)+C_{c f} C_{3} g_{m 2}+C_{m 1} C_{c f} g_{m 2} g_{m 3} \cdot\left(1 / g_{m c f}\right)}\right] \\
& \propto \frac{k_{1}}{k_{2}+\left[C_{c f} \cdot\left(k_{3}+k_{4} / g_{m c f}\right)\right]}
\end{aligned}
$$



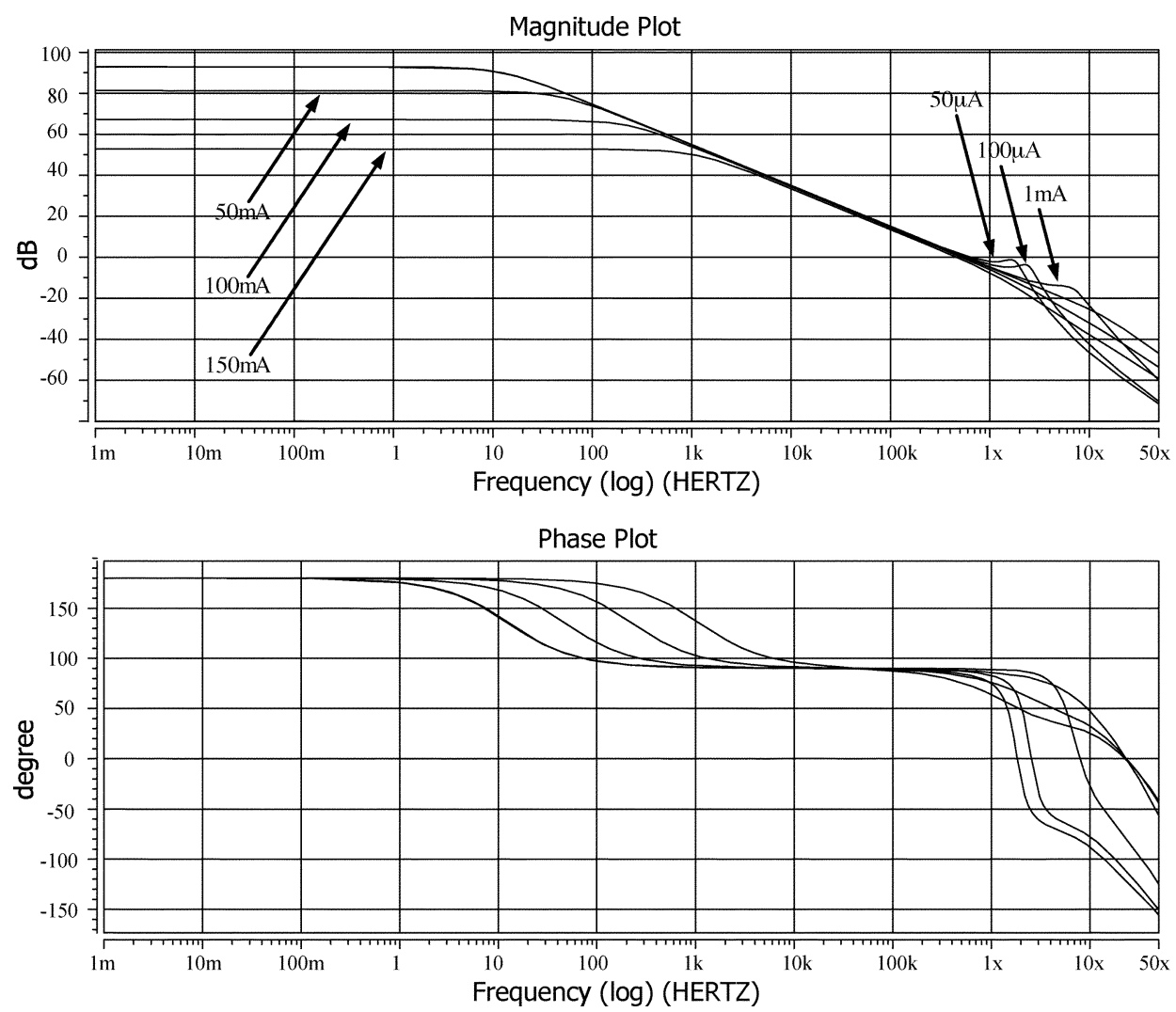

Fig. 6. Loop-gain simulation $\left(V_{\mathrm{IN}}=1.2 \mathrm{~V}\right.$ and $\left.V_{\text {OUT }}=1 \mathrm{~V}\right)$.

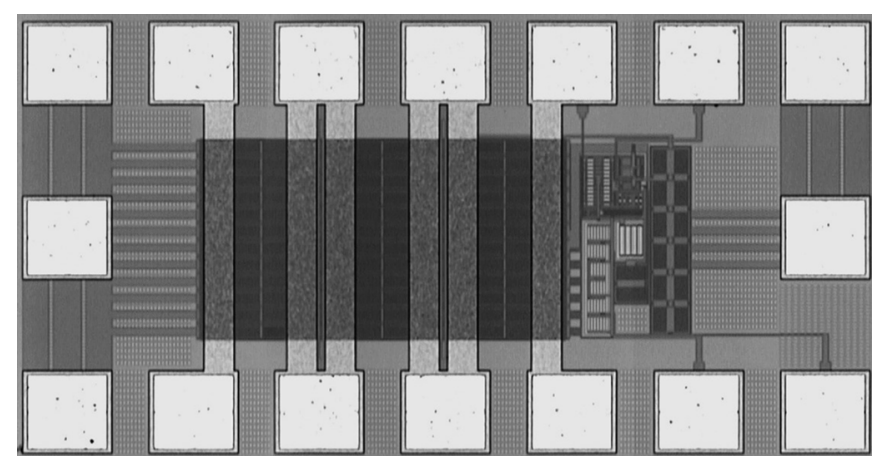

Fig. 7. Micrograph of proposed LDO (testing pads included).

bility. From the simulated results in Table I, the proposed LDO retains stable at different corners.

\section{EXPERIMENTAL RESULTS}

The proposed LDO has been implemented in AMS doublepoly triple-metal $0.35-\mu \mathrm{m}$ CMOS technology. The threshold voltages of nMOSFET and pMOSFET are $0.55 \mathrm{~V}$ and $-0.75 \mathrm{~V}$, respectively. The chip micrograph is shown in Fig. 7, and the active silicon area is $236 \mu \mathrm{m} \times 529 \mu \mathrm{m}$, excluding the testing pads. The LDO can operate down to $1.2 \mathrm{~V}$ with a preset output voltage of $1 \mathrm{~V}$. The dropout voltage at output current of $100 \mathrm{~mA}$ is $200 \mathrm{mV}$. The maximum ground current is about $100 \mu \mathrm{A}$ at $V_{\mathrm{IN}}=3.3 \mathrm{~V}$. The measured results are in Table II.

Load transient response with no off-chip capacitor (the estimated parasitic capacitance $C_{3}=100 \mathrm{pF}$ ) at $V_{\mathrm{IN}}=1.2 \mathrm{~V}$ and
TABLE I

CoRner-Model Simulations of THE Proposed LDO

Conditions: At $V_{D D}=1.2 \mathrm{~V}, V_{O}=1.0 \mathrm{~V}, I_{O U T}=100 \mu \mathrm{A}$

Temp $=25^{\circ} \mathrm{C}$
\begin{tabular}{|c|c|c|c|c|c|}
\hline Corners & tm & wo & wp & ws & wz \\
\hline UGF $(\mathrm{Hz})$ & $595 \mathrm{k}$ & $495 \mathrm{k}$ & $650 \mathrm{k}$ & $469 \mathrm{k}$ & $630 \mathrm{k}$ \\
\hline PM (degree) & 86.6 & 87.8 & 86.1 & 87.9 & 86.1 \\
\hline
\end{tabular}

Temp $=85^{\circ} \mathrm{C}$

\begin{tabular}{|c|c|c|c|c|c|}
\hline Corners & tm & wo & wp & ws & wz \\
\hline UGF (Hz) & $659 \mathrm{k}$ & $582 \mathrm{k}$ & $527 \mathrm{k}$ & $596 \mathrm{k}$ & $285 \mathrm{k}$ \\
\hline PM (degree) & 84.9 & 86.2 & 81.9 & 85.1 & 96.8 \\
\hline
\end{tabular}

Temp $=-40^{\circ} \mathrm{C}$
\begin{tabular}{|c|c|c|c|c|c|}
\hline Corners & tm & wo & wp & ws & wz \\
\hline UGF (Hz) & $514 \mathrm{k}$ & $355 \mathrm{k}$ & $588 \mathrm{k}$ & $335 \mathrm{k}$ & $575 \mathrm{k}$ \\
\hline PM (degree) & 88 & 89.5 & 87.6 & 89.8 & 87.5 \\
\hline
\end{tabular}

where $\mathrm{tm}=$ typical mean; $w o=$ worst one; $\mathrm{wp}=$ worst power;

$\mathrm{ws}=$ worst speed and $\mathrm{wz}=$ worst zero.

$V_{O}=1 \mathrm{~V}$ has been tested to verify the stability of the proposed LDO. As shown in Fig. 8, the LDO output voltage has no oscillation when the load current changes from $100 \mu \mathrm{A}$ to $100 \mathrm{~mA}$ in $1 \mu \mathrm{s}$, or vice versa. It is proven that the minimum output-current requirement for pole-splitting type LDO is reduced by the proposed $Q$-reduction circuit. Moreover, the proposed LDO is 

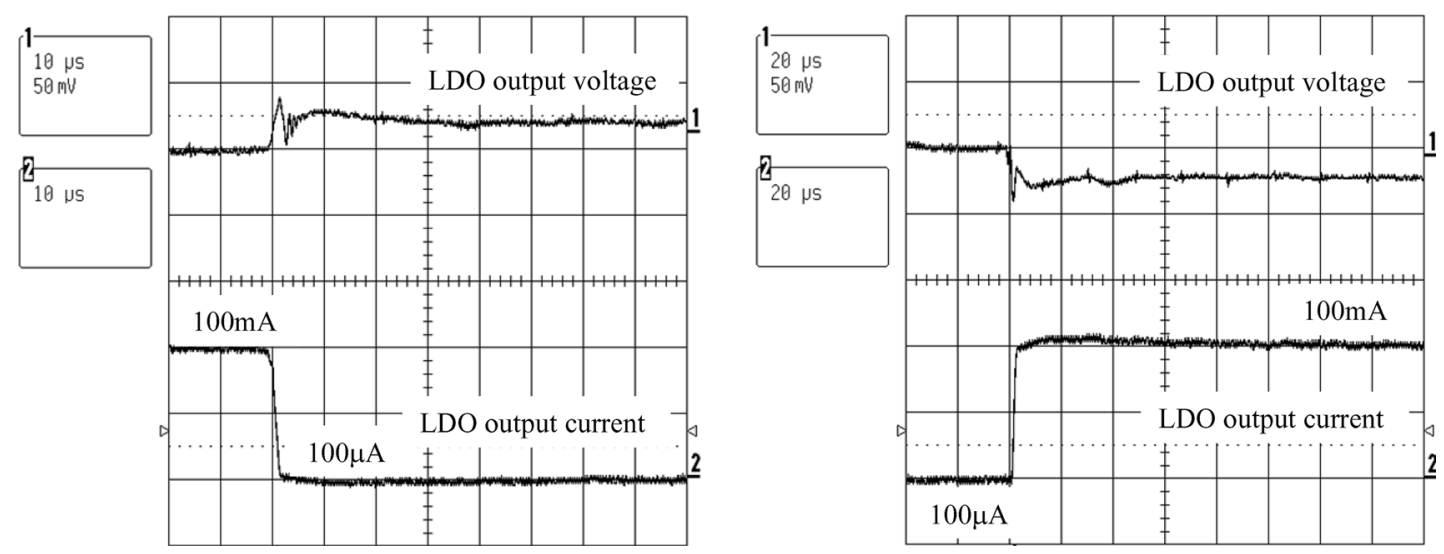

Fig. 8. Measured load transient responses at $V_{\mathrm{IN}}=1.2 \mathrm{~V}, V_{\mathrm{OUT}}=1 \mathrm{~V}, C_{3}=100 \mathrm{pF}$.
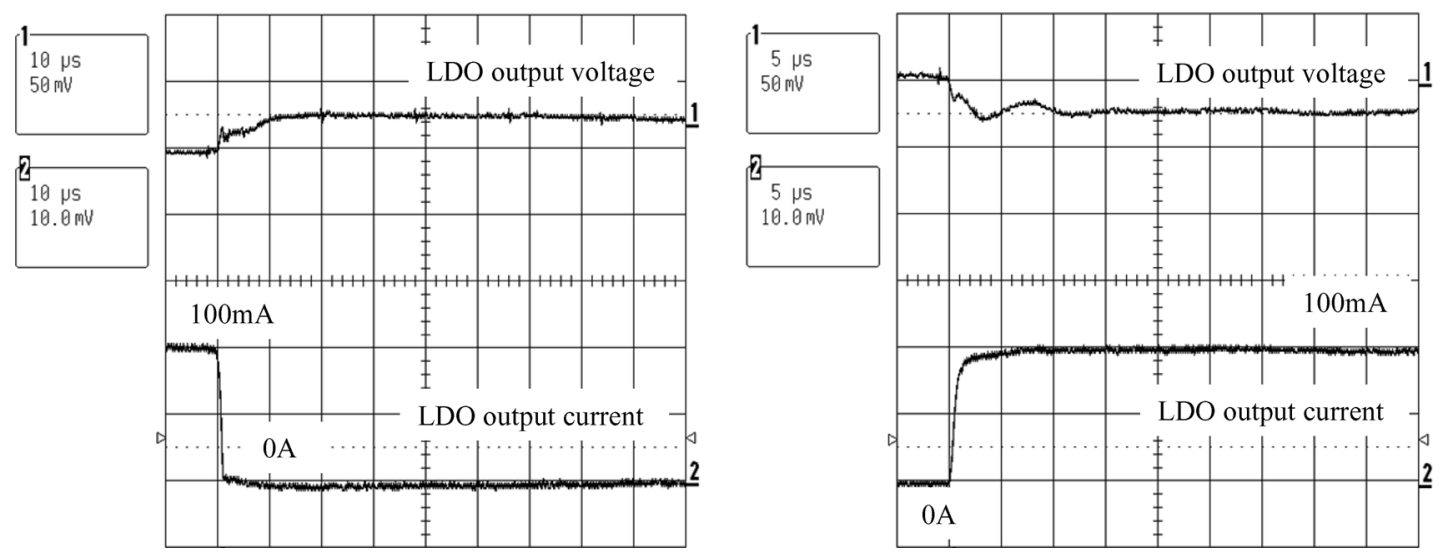

Fig. 9. Measured load transient responses at $V_{\mathrm{IN}}=1.2 \mathrm{~V}, V_{\mathrm{OUT}}=1 \mathrm{~V}, C_{L}=10 \mu \mathrm{F}$.

TABLE II

Performance Summary of THE PROPOSED LDO

\begin{tabular}{|c|c|}
\hline Technology & AMS 0.35- $\mu \mathrm{m}$ CMOS 2P 3M \\
\hline Threshold Voltage & $V_{T H N} \oplus 0.55 \mathrm{~V}$ and $\mid V_{T H P} \oplus 0.75 \mathrm{~V}$ \\
\hline Input Voltage $\left(V_{I N}\right)$ & $1.2 \mathrm{~V}$ to $3.3 \mathrm{~V}$ \\
\hline Preset Output Voltage $\left(V_{o}\right)$ & $1.0 \mathrm{~V}$ \\
\hline Output Current & $100 \mu \mathrm{A}$ to $100 \mathrm{~mA}$ \\
\hline Dropout Voltage & $200 \mathrm{mV} @ 100 \mathrm{~mA}$ \\
\hline Line regulation & $344 \mu \mathrm{V} / \mathrm{V} @ 100 \mathrm{~mA}$ \\
\hline Load Regulation & $-338 \mu \mathrm{V} / \mathrm{mA} @ V_{I N}=1.2 \mathrm{~V}$ \\
\hline On-chip capacitance & $C_{m l}+C_{c f}=6 \mathrm{pF}$ \\
\hline Power-line load capacitance modeling & $C_{3}=100 \mathrm{pF}$ \\
\hline Active Chip Area & $236 \mu \mathrm{m} \times 529 \mu \mathrm{m}$ (excluding testing pads) \\
\hline
\end{tabular}

also stable when an off-chip capacitor is used. The equivalent series resistance of the off-chip capacitor creates a LHP zero for pole-zero cancellation to achieve LDO stability. Fig. 9 shows the load transient responses when a $10-\mu \mathrm{F}$ off-chip capacitor is used.

\section{CONCLuSION}

A LDO for SoC has been introduced, supported by circuitmodeling analysis and experimental results. The proposed LDO structure and the proposed $Q$-reduction circuit, as well as its circuit implementation enable a smaller chip area and lower minimum output current requirement, which are very important for future $\mathrm{SoC}$ on chip, local voltage regulations.

\section{ACKNOWLEDGMENT}

The authors would like to thank S. F. Luk for his technical support.

\section{REFERENCES}

[1] D. D. Buss, "Technology in the internet age," in IEEE ISSCC Dig. Tech. Papers, 2002, pp. 18-21.

[2] G. A. Rincon-Mora, "Active capacitor multiplier in miller-compensated circuits," IEEE J. Solid-State Circuits, vol. 35, no. 1, pp. 26-32, Jan. 2000.

[3] K. N. Leung, P. K. T. Mok, and S. K. Lau, "A low-voltage CMOS lowdropout regulator with enhanced loop response," in IEEE Int. Symp. Circuits and Systems, 2004, vol. 1, pp. 385-388.

[4] C. K. Chava and J. Silva-Martinez, "A frequency compensation scheme for LDO voltage regulators," IEEE Trans. Circuits Syst. I, vol. 51, no. 6, pp. 1041-1050, Jun. 2004. 
[5] H. Pan, C. H. Cheng, and C. L. Chen, "A CMOS low dropout regulator stable with any load capacitor," in Proc. IEEE TENCON, 2004, pp. 266-269.

[6] P. Hazucha, T. Karnik, B. A. Bradley, C. Parsons, D. Finan, and S. Borkar, "Area-efficient linear regulator with ultra-fast load regulation," IEEE J. Solid-State Circuits, vol. 40, no. 4, pp. 933-940, Apr. 2005.

[7] D. Heisley and B. Wank, "DMOS delivers dramatic performance gains to LDO regulators," EDN Mag., pp. 141-150, Jun. 2000.

[8] K. N. Leung and P. K. T. Mok, "A capacitor-free CMOS low-dropout regulator with damping-factor-control frequency compensation," IEEE J. Solid-State Circuits, vol. 38, no. 10, pp. 1691-1702, Oct. 2003.

[9] —_ "Analysis of multi-stage amplifier-frequency compensation," IEEE Trans. Circuits Syst. I, vol. 48, no. 9, pp. 1041-1056, Sep. 2001.

[10] P. Gray, P. J. Hurst, S. H. Lewis, and R. G. Meyer, Analysis and Design of Analog Integrated Circuits, 4th ed. New York: Wiley, 2001.

[11] G. Palumbo and S. Pennisi, Feedback Amplifiers: Theory and Design. Norwell, MA: Kluwer Academic, Jan. 2002.

[12] _ - "Design methodology and advances in nested-Miller compensation," IEEE Trans. Circuits Syst. I, vol. 49, no. 7, pp. 893-903, Jul. 2002.

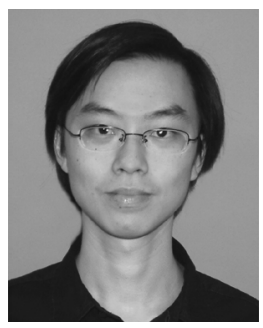

Sai Kit Lau (S'03-M'04) received the B.Eng. degree (First Class Honors) and M.Phil. degree in Electrical and Electronic Engineering from The Hong Kong University of Science and Technology, Hong Kong, in 2002 and 2004, respectively. His M.Phil. research areas include power-management integrated circuits in CMOS technology, frequency compensation topologies for multistage amplifiers and linear regulators.

Since 2004, he has been a Design Engineer with Solomon Systech Limited, Hong Kong. His areas of interests include power management system designs, analog integrated circuits of LCD and OLED driver controller ICs for mobile phones and handheld device applications. Currently, he is with Pericom Technology Inc., Hong Kong, as a Design Engineer working on the battery-charger controller ICs for batteryembedded devices.

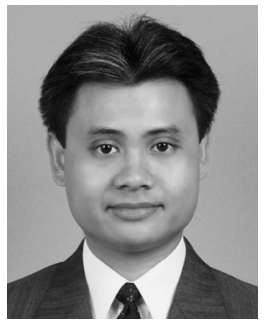

Philip K. T. Mok (S'86-M'95-SM'02) received the B.A.Sc., M.A.Sc., and Ph.D. degrees in electrical and computer engineering from the University of Toronto, Toronto, ON, Canada, in 1986, 1989 and 1995, respectively.

In January 1995, he joined the Department of Electrical and Electronic Engineering, The Hong Kong University of Science and Technology, Hong Kong, China, where he is currently an Associate Professor. His research interests include semiconductor devices, processing technologies and circuit designs for power electronics and telecommunications applications, with current emphasis on power management integrated circuits, low-voltage analog integrated circuits and RF integrated circuits design.

Dr. Mok received the Henry G. Acres Medal, the W.S. Wilson Medal and a Teaching Assistant Award from the University of Toronto, and the Teaching Excellence Appreciation Award twice from The Hong Kong University of Science and Technology. He is also a co-recipient of the Best Student Paper Award in the 2002 IEEE Custom Integrated Circuits Conference. In addition, he has been a member of the International Technical Program Committees of the IEEE International Solid-State Circuits Conference (ISSCC) since 2005 and he has served as an associate editor for IEEE TRANSACTIONS ON CIRCUITS AND SYSTEMS-II and IEEE JOURNAL OF SOLID-STATE CIRCUITS since 2006.

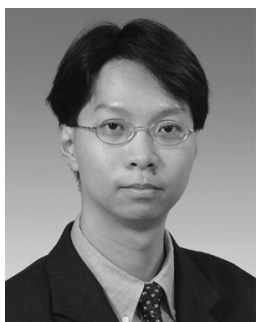

Ka Nang Leung (S'02-M'03) received the B.Eng., M.Phil., and Ph.D. degrees, all in electrical and electronic engineering, from The Hong Kong University of Science and Technology. During his B.Eng. studies, he joined Motorola, Hong Kong, to develop a PDA system as his final-year project. His Ph.D. research area was power-management integrated circuits in CMOS technology.

$\mathrm{He}$ joined the Department of Electronic Engineering, Chinese University of Hong Kong, in September 2005, as an Assistant Professor. He was a Visiting Assistant Professor in the Department of Electrical and Electronic Engineering, Hong Kong University of Science and Technology. He is a paper reviewer of several technical journals and international conferences. His current research interests include power-management integrated-circuit designs for telecommunication applications, $G m-C$ filter designs for bio-medical applications and CMOS image sensors.

In 1996, Dr. Leung received a Best Teaching Assistant Award from the Department of Electrical and Electronic Engineering, Hong Kong University of Science and Technology. He was the recipient of the 2003 Young Scientist Awards from the Hong Kong Institution of Science. 